\title{
Evaluación neuropsicológica, factores psicosociales y co-morbilidad psiquiátrica en pacientes pediátricos infectados con el VIH
}

\author{
Marta Castro P., Yaquelin Martínez V., Ida González N., Juana Velásquez A., Gladys Castillo I. y Lizet Sánchez V.
}

Instituto de Medicina Tropical "Pedro
Kouri" (MCP, IGN, LSV).
Hospital Pediátrico Docente Juan
Manuel Márquez (YMV, JVA, GCI).

Recibido: 9 de junio de 2010 Aceptado: 28 de marzo de 2011

Correspondencia a: Marta Castro Peraza martac@ipk.sld.cu

\section{Neuropsychological evaluation, psychosocial factors, and psychiatric comorbidity of children with HIV infection}

Antiretroviral therapy has marked a change in the course of HIV infection, posing new challenges to improve quality of life in pediatric patients. Objective: The goal of this paper was to identify psychosocial aspects of HIV-infected children and adolescents, and to evaluate their neuropsychological development and psychiatric comorbidity. Patients and Methods: A descriptive study was conducted, evaluating the neurocognitive performance of patients attending at the Pediatric Department of Instituto de Medicina Tropical Pedro Kourí of La Habana, Cuba, between February and July of 2008. For that purpose, interviews to the patients and their relatives were done with the application of appropriate tests for each age. Results: Twenty-three patients ages 3-17 years (57\% female) were studied. Sixteen (69.5\%) were infected through vertical transmission, and 7 (30.5\%) through other routes. School children infected through vertical transmission showed the most symptoms. Hyperactivity, anxiety, and learning disorders were the most frequently observed symptoms as well as hyperkinetic disorders, temporary tic disorders and mental retardation. Conclusion: This study shows the neuropsychological characterization of HIV-positive children and adolescents, considering the route of acquisition of the infection and the stage of the disease. It also describes psychiatric comorbidity of HIV-infected children.

Key words: HIV/AIDS, psychosocial, neuropsychological disorders, psychiatric comorbidity, family, Cuba.

Palabras clave: VIH, síndrome de inmunodeficiencia humana, psicosocial, trastornos neuropsicológicos, comorbilidad psiquiátrica, Cuba.

\section{Introducción}

$\mathrm{E}$ n el mundo, 2 millones de niños estaban infectados por el virus de la inmunodeficiencia humana en el año 2008; aproximadamente $45 \%$ de las nuevas infecciones ocurrieron en personas jóvenes entre 15 y 24 años $^{1}$.

Sin tratamiento específico, la mitad de los niños con exposición prenatal fallecen en los primeros años de la vida. Al igual que en el adulto, el tratamiento en las edades pediátricas ha experimentado notables cambios en los últimos años hacia una intervención más precoz y con terapias combinadas más potentes ${ }^{2-6}$. El tratamiento anti-retroviral (TARV) ha supuesto un cambio radical en la evolución de los niños infectados por el VIH, transformando la evolución, de ser una enfermedad mortal a corto plazo, en una elevada proporción de casos, a seguir una evolución crónica. Por lo que en la actualidad se plantean nuevos retos frente a la infección por el virus y las complicaciones asociadas a la terapéutica.

El Programa de Prevención y Control del VIH/SIDA en Cuba incorporó desde el año 1986 medidas profilácticas para la trasmisión vertical ${ }^{7,8}$. El cumplimiento de estas normativas establecidas por el programa ha contribuido a que hasta el 31 de diciembre del año 2007 se pesquisara un total de 314 niños hijos de madres con infección por VIH/SIDA. Completaron su seguimiento 248 y de ellos, 32 resultaron infectados ${ }^{9-11}$.

Entender el origen de las alteraciones neuropsicológicas que se presentan en el curso de la infección por el VIH ha sido objeto de múltiples estudios desde los inicios de la epidemia. Evidencias reconocen que la infección se asocia con un riesgo mayor de daño al sistema nervioso central (SNC), considerándose que entre 15 y 25\% de los niños podían presentar un daño grave y, a menudo, manifestaciones progresivas de lesión. En ausencia del TARV, la encefalopatía asociada al VIH se presenta frecuentemente antes del año de vida, comprometiendo el neurodesarrollo y la calidad de vida del niño. Se ha demostrado que el déficit neurocognitivo puede presentar un curso variable, teniendo en cuenta el estado inmunológico del paciente, lo que se modifica con el uso del TARV ${ }^{3-5,12}$.

Sin embargo, las alteraciones en el comportamiento en las edades pediátricas no sólo están determinadas por los 
efectos directos causados por la infiltración del cerebro; otros factores no menos importantes pueden jugar un papel fundamental en la patogenia de estos trastornos. Factores vinculados al ambiente familiar, condiciones socio-demográficas, experiencias traumáticas dependientes de la práctica de múltiples procedimientos médicos y hospitalizaciones, el rechazo social y la limitación del juego con pares, por citar las más frecuentes ${ }^{4,12}$.

Cuba cuenta con una estrategia de respuesta a la infección por VIH/SIDA, con un enfoque integral que garantiza y protege los derechos de las personas con VIH/SIDA. El enriquecer y perfeccionar las prácticas actuales, con un enfoque integral y multidisciplinario de la atención al paciente en edad pediátrica, que incorpore elementos de promoción y prevención de los trastornos neuropsicológicos, puede contribuir al aumento sostenible de la calidad de vida.

El presente estudio se propuso como objetivos identificar aspectos psicosociales en niños y adolescentes que adquirieron la infección por el VIH en Cuba, evaluar su desarrollo neuropsicológico y describir la co-morbilidad psiquiátrica presente en el grupo estudiado.

\section{Material y Métodos}

Se realizó un estudio de tipo descriptivo. El universo lo constituyó la cohorte de 30 niños que adquirieran la infección de las siguientes maneras: por transmisión vertical (n: 22), uso de sangre y hemoderivados (n: 3) e inicio de actividad sexual en adolescentes (n: 5), todos vivos y en control periódico en el Instituto de Medicina Tropical Pedro Kourí mientras se realizó la investigación. La población estudiada quedó constituida por 23 pacientes que asistieron a la consulta especializada de pediatría en el período comprendido entre los meses de febrero y julio de 2008, procedentes de todas las provincias del país.

Procedimientos. En todos los casos se realizó, previa al inicio del estudio, una re-evaluación clínica por el equipo de atención pediátrica, la cual se tuvo en cuenta en la evaluación integral de los participantes. La evaluación fue conducida en dos sesiones. En un primer momento se realizó entrevista estructurada a familiares que están al cuidado del niño, donde se exploraron variables sociodemográficas (sexo, edad, color de la piel, nivel educacional y convivencia familiar), presencia de síntoma psicológicos teniendo en cuenta el desempeño en el área escuela, calidad de las relaciones con sus iguales y en el área familiar. Se utilizaron las historias clínicas para caracterizar la enfermedad recogiéndose los datos: vías de adquisición de la infección, edad al diagnóstico y clasificación clínica de la infección al momento de realizarse el estudio.
La clasificación clínica del paciente se realizó según lo normado por el CDC de Atlanta, E.U.A.; se empleó dos categorizaciones, la de $1987^{13}$, que se aplicó a los niños nacidos hasta 1996 teniendo en cuenta sólo el estado clínico e infecciones experimentadas, (en Cuba no se contaba, hasta esa fecha, con el citómetro de flujo para realizar sub-población linfocitaria CD3, CD4, y CD8), y la de $1994^{14}$, utilizada a partir de entonces, en la que se reclasificaron los niños vivos y los nuevos nacimientos teniendo en cuenta el estado clínico e inmunológico del niño.

En un segundo tiempo, se realizó el examen psiquiátrico directo en cada caso estudiado y se realizaron tests psicométricos para la evaluación del desarrollo neuropsicológico. Cuba tiene experiencia en la evaluación pediátrica con los tests que fueron incluidos por los autores, tanto en el sistema nacional de salud como en el sistema nacional de educación, con más de 20 años de experiencia. En todos los casos se tuvo en cuenta la edad cronológica para la selección de las pruebas y se respectaron las condiciones requeridas para la aplicación de cada test, que se relacionan a continuación ${ }^{16,17}$.

Instrumentos utilizados para la evaluación neurocognitiva:

- De 3-7 años: Terman Merrill y el test gestáltico visomotor de Lauretta Bender.

- De 8-12 años: La escala de Wechsler (WISC) $1^{\text {a }}$ edición, para las edades de 8-17 años, el test de tachado de letras (test de atención de R. H. Crespo), el test de lateralidad (prueba de R. Zazzo), el cuarto excluido, la metódica de memoria de Luria (10 palabras de A. N. Luria) y el test gestáltico visomotor de Lauretta Bender.

- De 13-15 años: La escala de Wechsler (WISC) $1^{\text {a }}$ edición, para las edades de 8-17 años y el test de atención de tachado de letras (test de atención de R. H. Crespo).

- De 16-19 años: La escala de Wechsler (WAIS) $1^{\text {a }}$ edición para edades superiores a los 17 años, el Machover y el cuarto excluido.

En todos los participantes se exploró la presencia de un diagnóstico psiquiátrico parametrado, para lo cual se utilizó como referencia los criterios de la Décima Clasificación Internacional de Enfermedades en su Manual de Clasificación de los Trastornos Mentales y del Comportamiento: Descripciones clínicas y pautas para el diagnóstico (CIE-10) ${ }^{17}$. El pediatra formó parte del equipo de evaluación y sus observaciones e intereses se tomaron en cuenta en la conclusión de la misma. En todos los casos se realizaron intervenciones psicológicas de acuerdo a los resultados de la evaluación y las necesidades de atención identificadas por pacientes familiares.

Análisis estadístico. Se creó una base de datos en 
Tabla 1. Características sociodemográficas de los niños portadores de infección por VIH/SIDA y familiares participantes. (n: 23) Instituto de Medicina Tropical Pedro Kourí, La Habana, Cuba

\begin{tabular}{lcc} 
Características & n & $\%$ \\
Sexo & 13 & 56,5 \\
Femenino & 10 & 43,5 \\
Masculino & & \\
Color de la piel & 14 & 60,9 \\
Blanco & 5 & 21,7 \\
Negro & 4 & 17,4 \\
Mestizo & & \\
Edad & 7 & 30,4 \\
Prescolares (3-5años) & 10 & 43,5 \\
Escolares (6-11 años) & 6 & 26,1 \\
Adolescentes (12-17 años) & & \\
Nivel escolar actual & 5 & 21,7 \\
No inician escolarización & 12 & 52,2 \\
Primaria & 3 & 13,0 \\
Secundaria & 1 & 4,4 \\
Técnico medio o preuniversitaria & 2 & 8,7 \\
No vinculo actual & & \\
Familiares convivientes & 12 & 52,2 \\
Madre & 2 & 8,7 \\
Padre & 5 & 21,7 \\
Ambos padres & 4 & \\
Otros tutores & 5,4 \\
\hline
\end{tabular}

Tabla 2. Descripción de la infección por VIH/SIDA en niños y adolescentes estudiados. Instituto de Medicina Tropical Pedro Kourí, La Habana, Cuba

\section{Características}

\section{Edad del diagnóstico}

$<1$ año

1-4 años

5-9 años

10-14 años

15-18 años

Vía de adquisición de la infección

Vía vertical

Otras vías

\section{Clasificación clínica}

A

B

C

\section{TARV}

Con tratamiento

Sin tratamiento n

$\%$

$\begin{array}{rc}3 & 13,0 \\ 13 & 56,6 \\ 2 & 8,7 \\ 3 & 13,0 \\ 2 & 8,7\end{array}$

16

12

5

6

17

6
Excel; para hacer los cálculos correspondientes se utilizó el paquete estadístico SPSS versión 11.5. Se realizaron los análisis de frecuencia simples de las variables sociodemográficas estudiadas, la evolución clínica de la infección, síntomas observados y diagnósticos parametrados. Se calcularon las medias para la ponderación global de los coeficientes de inteligencia para escala de Weschler: coeficiente intelectual (CI) y edad mental (EM) para el test de Terman Merrill; además se cálculo el rango de los valores para ambas escalas utilizadas. Se compararon los porcentajes de síntomas identificados teniendo en cuenta las etapas del desarrollo y síntomas identificados, utilizando la prueba $\chi^{2}$ de comparación de proporciones. Se consideró un nivel de significación inferior de 0,05.

Consideraciones éticas. Se incluyeron los pacientes que voluntariamente desearon incorporarse después de explicarles a ellos, sus padres o tutores, los objetivos de la investigación. En el estudio, por incluir niños menores de 18 años, se pidió el consentimiento informado al familiar o tutor que lo representaba, aunque en todos los casos se les solicitó aquiescencia a los niños para trabajar con ellos, y en caso de los adolescentes su negación, aun cuando el familiar lo aceptara, sería excluyente. Se aseguró la asistencia especializada, por un equipo de salud mental a todo niño que formó parte del estudio y que solicitara atención o reuniera criterios para ello.

\section{Resultados}

Concluyeron el estudio y se consideraron en el análisis un total de 23 niños y adolescentes, entre las edades de 3 y 17 años. Como se muestra en la Tabla 1, predominó el sexo femenino con 13 (56,5\%), el color de la piel blanca en 14 (60,8\%) y las edades entre 6-11 años en 10 (43,5\%). Más de $50 \%$ de los niños se encontraban en los primeros años de escolarización o nivel primario y sólo en cinco $(21,7 \%)$ participantes se recogió el antecedente de convivir con ambos padres.

La edad más frecuente con que se realizó el diagnóstico en este grupo fue entre 1-4 años (n: 13; 56,6\%); sólo en un niño infectado por vía vertical el diagnóstico se realizó después de estas edades. La vía de adquisición que predominó fue la vertical (n: 16; 69,5\%). El estudio incluyó adolescentes que se infectaron por otras vías, cinco por la vía sexual y uno por transfusión de sangre y hemoderivados. De acuerdo a la evolución clínica de la enfermedad, predominó el grupo A (n: 12; 52,1\%), seguido de los grupos B (n: 5; 21,8\%) y C (n: 6; 26,1\%), respectivamente. En el momento en que se realizó el estudio tenían indicado TARV 18 (72,8\%) de los participantes (Tabla 2).

En la Tabla 3 se muestran los síntomas constatados al 
Tabla 3. Síntomas constatados al examen psiquiátrico de pacientes pediátricos con infección por VIH/SIDA, según etapas del desarrollo. Instituto de Medicina Tropical Pedro Kourí, La Habana, Cuba. (n: 23)

\begin{tabular}{|c|c|c|c|c|c|c|c|}
\hline \multirow[t]{3}{*}{ Sintomatología observada } & \multicolumn{6}{|c|}{ Etapas del desarrollo } & \multirow{3}{*}{ valor $\mathbf{p}$} \\
\hline & \multicolumn{2}{|c|}{$\begin{array}{c}\text { Preescolares } \\
n=7\end{array}$} & \multicolumn{2}{|c|}{$\begin{array}{l}\text { Escolares } \\
n=10\end{array}$} & \multicolumn{2}{|c|}{$\begin{array}{c}\text { Adolescentes } \\
\qquad n=6\end{array}$} & \\
\hline & $\mathbf{n}$ & $\%$ & $\mathbf{n}$ & $\%$ & $\mathbf{n}$ & $\%$ & \\
\hline Hiperactividad & 4 & 57,1 & 5 & 50,0 & 0 & 0 & 0,07 \\
\hline Ansiedad & 3 & 42,8 & 4 & 40,0 & 0 & 0 & 0,16 \\
\hline Dificultades de aprendizaje & 1 & 14,2 & 6 & 60,0 & 3 & 50,0 & 0,16 \\
\hline Perretas & 1 & 14,2 & 2 & 20,0 & 0 & 0 & 0,51 \\
\hline Irritabilidad & 1 & 14,2 & 1 & 10,0 & 1 & 16,6 & 0,92 \\
\hline Impulsividad & 1 & 14,2 & 3 & 30,0 & 2 & 33,3 & 0,68 \\
\hline Alteraciones del sueño & 2 & 28,6 & 1 & 10,0 & 1 & 16,6 & 0,61 \\
\hline Tristeza & 0 & 0 & 1 & 10,0 & 5 & 83,3 & $<0,000$ \\
\hline Timidez & 0 & 0 & 1 & 10,0 & 1 & 16,6 & 0,55 \\
\hline Anorexia & 1 & 14,2 & 1 & 10,0 & 1 & 16,6 & 0,92 \\
\hline Enuresis & 0 & 0 & 2 & 20,0 & 0 & 0 & 0,24 \\
\hline
\end{tabular}

examen psiquiátrico según la etapa del desarrollo. Los escolares fueron quienes presentaron mayor sintomatología, observándose hiperactividad en cinco niños estudiados (50,0\%), ansiedad en cuatro $(40,0 \%)$ y dificultades en el aprendizaje en seis (60,0\%), los cuales fueron referidos también por parte de los padres. La tristeza fue el síntoma que predominó en la adolescencia, lo cual resultó estadísticamente significativo y donde la vía sexual de adquisición de la infección fue la más importante.

La Tabla 4 muestra el desempeño de los niños en los tests neuro-cognitivos. Todos los niños pudieron ser evaluados con un test de inteligencia de acuerdo a la edad cronológica; a 14 pacientes se le realizó el test de medición de la inteligencia de Terman Merrill. La media de las puntuaciones del coeficiente de inteligencia fue de 90,4 con un rango entre 69 y 100. El déficit cognitivo sólo pudo ser constatado en un caso con un retraso mental ligero. En seis niños se utilizó la escala de Weschler (WISC); la media de los valores del CI fue de 84,6 y las puntuaciones estuvieron en un rango entre 81 y 88. A tres adolescentes se le aplicó la escala de Weschler (WAIS), encontrándose una media de la puntuación total de CI de 85,6; el rango de los valores estuvo entre 90 y 84 .

En la metódica de pensamiento (Cuarto excluido) dos de los niños en edad escolar, a los que se les realizó esta prueba, no pudieron realizar correctamente la conceptualización.

En la metódica de memoria (10 palabras de A.N. Luria) en cinco niños se observó la presencia de una alteración de la memoria a corto y largo plazo.

En el test de atención (tachado de letras de R.H.
Tabla 4. Resultados de los exámenes para la evaluación neuropsicológica. Instituto de Medicina Tropical Pedro Kourí, La Habana, Cuba

\begin{tabular}{|c|c|c|}
\hline Exámenes psicométricos & & \\
\hline Test de inteligencia & Media & Rango \\
\hline Terman Cerril & 90,4 & $69-100$ \\
\hline WISC & 84,6 & $81-88$ \\
\hline WAIS & 85,6 & $82-90$ \\
\hline Test de tachado de letras & & \\
\hline (Test de atención de R.H. Crespo) & $\mathrm{n}$ & $\%$ \\
\hline Déficit atentito & 3 & 20,0 \\
\hline Sin déficit atentito & 12 & 80,0 \\
\hline Metódica de memoria de Luria & & \\
\hline (10 palabras de A.N Luria) & $\mathrm{n}$ & $\%$ \\
\hline Afectación & 5 & 55,6 \\
\hline No Afectación & 4 & 44,4 \\
\hline Metódica de pensamiento & & \\
\hline (cuarto excluido) & $\mathrm{n}$ & $\%$ \\
\hline Alteración & 2 & 22,2 \\
\hline No alteración & 6 & 66,6 \\
\hline Test de lateralidad & & \\
\hline (Prueba de R. Zazzo) & $\mathrm{n}$ & $\%$ \\
\hline Definida & 9 & 100 \\
\hline No Definida & 0 & 0 \\
\hline Test de coordinación visomotora & & \\
\hline & $\mathrm{n}$ & $\%$ \\
\hline Con afectación & 2 & 28,6 \\
\hline Sin afectación & 5 & 71,4 \\
\hline
\end{tabular}


Tabla 5. Diagnósticos psiquiátricos en los niños y adolescentes estudiados, según criterios de la Décima Clasificación de Enfermedades (CIE-10)

\begin{tabular}{lcc} 
Diagnóstico Psiquiátrico & $\mathbf{n}$ & $\mathbf{\%}$ \\
\hline Trastorno hipercinético & 3 & 13,0 \\
\hline Trastorno de adaptación depresivo & 2 & 8,6 \\
\hline Trastorno no orgánico del sueño ("pesadilla") & 2 & 8,6 \\
\hline Retraso mental ligero & 1 & 4,3 \\
\hline Trastorno de tics transitorio & 1 & 4,3 \\
\hline Trastorno disocial & 1 & 4,3 \\
\hline Enuresis no orgánica & 1 & 4,3 \\
\hline Total & 11 & 47,8 \\
\hline
\end{tabular}

Crespo) encontramos que en $20,0 \%$ hay un déficit de atención.

Mientras que el test de lateralidad (prueba de R. Zazzo) realizado a nueve niños no mostró alteraciones.

El test gestáltico visomotor de Lauretta Bender se realizó en siete niños, encontrando que sólo dos pacientes presentaron afectación de esta función.

Como se muestra en la Tabla 5, fue posible realizar algún diagnóstico en 11 de los niños y adolescentes participantes. El trastorno hipercinético se consideró en tres pacientes y los trastornos de adaptación con síntomas depresivos y ansiosos, en dos adolescentes, estando relacionado en ambos casos con la notificación de la infección. El resto de los diagnósticos lo constituyeron, dos pacientes en edad pre escolar con trastornos no orgánicos del sueño (parasomnia), el trastorno de tics transitorio, retraso mental ligero, el trastorno disocial y la enuresis no orgánica, en un caso respectivamente.

\section{Discusión}

El estudio logró evaluar características del neurodesarrollo y llegar al diagnóstico de trastornos mentales prevalentes. Las alteraciones en las emociones fueron predominantes en la adolescencia, relacionada con la adquisición de la infección por vía de las relaciones sexuales. En los niños que adquirieron la infección por transmisión vertical, fueron de mayor importancia los trastornos del comportamiento, siendo el trastorno hipercinético y las dificultades relacionadas con el aprendizaje escolar, los de mayor protagonismo.

El estudio tuvo limitaciones que deben ser consideradas: la existencia de factores medio-ambientales como la exposición a TARV prenatal, la interacción madre-niño durante la primera etapa de la vida, los cambios en los cuidadores y particularidades del ambiente familiar que están jugando un papel importante en la sintomatología presente, no pueden ser valorados al incluirse solamente niños infectados por VIH. El grupo de estudio tiene una gran dispersión de sus edades lo cual dificultó el análisis por grupo de edades o etapas del desarrollo.

En Cuba, a los niños infectados por VIH se les chequean su estado clínico, inmunológico y virológico hasta estabilizarlos desde estos puntos de vista, en una consulta especializada. Además reciben una dieta especial rica en proteínas, grasas y carbohidratos y se les garantiza el acceso al TARV una vez que se decida por la evolución clínica de la enfermedad. En nuestro estudio, seis niños actualmente no tomaban TARV, pues se mantenían asintomáticos y no clasificaban como SIDA.

De acuerdo a las vías de adquisición de la infección, la sintomatología predominó en escolares infectados por vía vertical, donde se realizaron los diagnósticos de trastorno hipercinético, trastorno de tics transitorio y retraso mental ligero. Este último, relacionado con daño cerebral temprano y el diagnóstico de una parálisis cerebral, donde una posible explicación podría estar relacionada con el efecto de la disfunción neuropsicológica causada por la enfermedad.

El trastorno hipercinético, donde predomina la hiperactividad y la inatención, constituye otro de los trastornos frecuentemente encontrado en la literatura revisada. Su asociación al diagnóstico de infección por VIH/SIDA ha sido establecido en valores de $24,6 \%^{19}$, considerando que no sólo la infección por VIH juega un papel en su patogenia, sino que existen otros elementos que deben ser valorados ${ }^{5,19-21}$. En el estudio fue posible realizar el diagnóstico del trastorno hipercinético, pero vale resaltar que la hiperactividad como síntoma predominante resultó frecuentemente reportada y fue importante su interferencia en el ambiente familiar y escolar. En los escolares, es importante tener en cuenta que las características psicológicas propias de esta etapa son un reto para el diagnóstico de las alteraciones del estado de ánimo, lo cual debe ser tenido en cuenta por los equipos de asistencia.

Los trastornos predominantes en la adolescencia fueron las alteraciones emocionales, las que se relacionaron con la vía de transmisión sexual. Fue posible diagnosticar, en al menos dos casos, un trastorno de adaptación con síntomas depresivos, pero la tristeza fue un síntoma frecuente en estas edades. En los niños, la muerte de las figuras parentales a causa de la infección, puede ser un factor importante para que se estructuren síntomas depresivos como ha sido reportado por otros estudios ${ }^{19,20}$; en nuestro trabajo también fue posible constatarlo al coincidir el fallecimiento de un padre en el momento de estar realizándose la observación clínica.

Estudios longitudinales que incluyeron tamaños de muestra grandes hacen pensar que los niños infectados tienen un riesgo mayor para desarrollar sintomatología ansiosa, depresiva u otros problemas de comportamiento ${ }^{4,22}$. Para los niños y adolescentes se han reportado 
prevalencias entre 14 y 44\%, lo cual coincidió con este trabajo, para las alteraciones del comportamiento y de las emociones, que incluyen la ansiedad, la depresión y otros trastornos de conducta, pero estos reportes pueden variar en los diferentes contextos y tipos de diseño utilizados ${ }^{19,21-23}$.

La intervención temprana y oportuna en los niños $\mathrm{y}$ adolescentes que tenga en cuenta las necesidades individuales del niño y del ambiente familiar debe constituir una prioridad en la atención al paciente pediátrico infectado por VIH. El seguimiento de funcionamiento neuropsicológico puede aportar elementos en la evolución de la enfermedad; en todos los participantes la evaluación clínica fue un elemento esencial antes de realizar la evaluación neuropsicológica.

La vinculación en programas de apoyo psicosocial y la rehabilitación psicopedagógica pueden contribuir a una mejora significativa en la calidad de vida ${ }^{24}$. Otros trabajos que han abordado la problemática de los convivientes y cuidadores de personas infectadas mostraron que los familiares cuidadores desarrollaron sus propios modelos para cuidar a los niños con infección por VIH. De ellos, el más importante fue el miedo a la estigmatización; esto, a su vez, puede estar relacionado con la sintomatología exhibida y la percepción de necesidad de ayuda especializada ${ }^{25-28}$.

El trabajo aportó elementos en la evaluación del paciente pediátrico infectado por VIH teniendo en cuenta: las vías de adquisición de la infección, la visión de un grupo multidisciplinario que comprendió la vinculación de pediatras y especialistas en salud mental, lo cual permitió la caracterización neuropsicológica de los niños y adolescentes estudiados, teniendo en cuenta las vías de adquisición del infección, el momento evolutivo de la enfermedad y determinar la co-morbilidad asociada. El seguimiento de la corte estudiada pudiera aportar nuevas evidencia a la evolución neuropsicológica en el curso de la infección por VIH en estas etapas de la vida.

Agradecimientos: A los niños y niñas, a los adolescentes y las adolescentes, a sus familias los cuales forman parte de las personas que hoy viven infectados por VIH en Cuba y que contribuyeron a este estudio desde sus experiencias de vida. La investigación fue financiada por el Ministerio de Salud Pública de Cuba.

\section{Resumen}

El tratamiento anti-retroviral marcó un cambio en la evolución de la infección por VIH, planteándose nuevos retos que mejoren la calidad de vida en las edades pediátricas. Objetivos: Identificar aspectos psicosociales en niño y adolescente que adquirieron la infección por VIH, evaluar el desarrollo neuropsicológico y describir la co-morbilidad psiquiátrica presente en el grupo estudiado. Pacientes y Métodos: Se realizó un estudio descriptivo, donde se evaluó el desempeño neurocognitivo de pacientes atendidos en la clínica pediátrica del Instituto de Medicina Tropical Pedro Kourí de La Habana, Cuba, entre los meses de febrero y julio de 2008, para lo cual se realizaron entrevistas a familiares, examen directo del participante y la aplicación de instrumentos específicos para cada edad. Resultados: Se evaluaron 23 niños y adolescentes entre los 3 y 17 años, predominó el sexo femenino con $13(56,5 \%)$ y las edades entre los 6-11 años con 10 (43,5\%) niños, más del 50\% de los cuales se encontraban en los primeros años de escolarización, 16 $(69,5 \%)$ infectados por trasmisión vertical y 7 (30,5\%) por otras vías. Los escolares infectados por vía vertical fueron los que mayor sintomatología aportaron, la hiperactividad $(57,1 \%)$, la ansiedad (42,8\%) y las dificultades en el aprendizaje (14,2\%), fueron los síntomas más frecuentes. El diagnóstico de trastorno hipercinético (13,0\%) fue el más importante en la etapa escolar, mientras que el trastorno de adaptación depresivo lo fue en la adolescencia (8,6\%). Conclusión: El estudio permitió la caracterización neuropsicológica de los niños y adolescentes estudiados, teniendo en cuenta las vías de adquisición de la infección, el momento evolutivo de la enfermedad y describir la co-morbilidad psiquiátrica asociada.

\section{Referencias}

1.- $\quad$ AIDS Epidemic Update. Geneva: UNAIDS; 2009. Disponible en: http://www.unaids.org/ en/HIV_data/epi2009/ (Accedido: 4 de abril de 2010).

2.- Brouwers P, Moss H, Wolters P, Eddy J, Balis F, Poplack D G, et al. Effect of continuous infusion zidovudine therapy o neuropsychological functioning in children with symptomatic immunodeficiency virus infection. J Pediatr. 1990; 117: 980-5.
3.- Culnane M, Fowler M, Lee SS, McSherry G, Brady M, O’Donnell K, et al. Lack of long term effect of in utero exposure to zidovudine among uninfected children born to HIV infected women. JAMA 1999; 281: 151-7.

4.- Jeremy R J, Kim S, Nozyce M, Nachman S, McIntosh K, Pelton S I, et al. Neuropsychological functioning and viral load in stable antiretroviral therapy-experienced HIV-infected children. Pediatrics. 2005; 115: 380-387.

5.- Lindsey J C, Malee K M, Brouwers P, Michael D, Hughes M D, for the PACTG 219C Study Team. Neurodevelopmental functioning in HIV-infected infants and young children before and after the introduction of protease inhibitor-based highly active antiretroviral therapy. Pediatrics 2007; 119: e681-93.

6.- Llorente A, Brouwers P, Charurat M, Magder L, Malee K, Mellins C, et al. Women and infant transmission study group. Early neurodevelopment markers predictive of mortality in infants infected with HIV-1. Dev Med Chil Neurol 2003; 45: 76-84.

7.- $\quad$ Ministerio de Salud Pública. Plan estratégico nacional ITS/VIH/Sida, 2001-2006. La Habana (Cuba): Ministerio de Salud Pública; Ene 2001. 
8.- Ministerio de Salud Pública, Dirección Nacional de Epidemiología. Programa Nacional de Prevención y control del VIH/SIDA. Actualización. La Habana (Cuba): Ministerio de Salud Pública; Abril 1997.

9.- González I, Díaz J, Verdasquera D, Pérez L. Prevention and control of vertical HIV transmission program in Cuba: January 1986December 2007. Rev Chil Infectol [online]. 2010, vol.27, n.4, pp. 320-6.

10.- González I, Jidy M, Pérez J. Vertical transmission in Cuba. MEDICC Review 2006; 8(1). Disponible en: http//www.medicc.org/ publications/medicc_review/0406/cubanmedical-literature.html (Accedido: 16 de mayo de 2007).

11.- González I, Jidy M, Berdasquera D, Berdasquera D. Toledo M E, Pérez J. Infección por VIH en Cuba por transmisión vertical. Reporte de nueve casos fatales en 10 años VIH/SIDA en edades pediátricas, Cuba 1986-2004. Rev Chil Infectol 2008; 25 (1): 41-8.

12.- Coscia J M, Chistensen B K, Henry R, Wallston K, Radcliffe J, Rutstein R. Effects of home environment, socioeconomic status, and health status on cognitive functioning i children with HIV-1 Infection. J Ped Psych 2001; 6: 321-9.

13.- Centers for Diseases Control and Prevention. Revision of the surveillance case definition for acquired immunodeficiency syndrome. MMWR Morbid Mortal Wkly Rep 1987; 36 (Suppl 15): 15-135.

14.- Centers for Diseases Control and Prevention.
1993 revised classification system for human immunodeficiency virus infection in children less than 13 years of age. MMWR Morbid Mortal Wkly Rep 1994; 43 (RR-12): 1-15.

15.- González F M. Instrumentos de evaluación Psicológica. La Habana: Editorial Ciencias médica; 2007. http://gsdl.bvs.sld.cu/PDFs/ Coleccion_de_Psicologia/inst_eval_psico/ completo.pdf

16.- Rodríguez O. Salud Mental Infanto-Juvenil. La Habana: Editorial Ciencias Médicas; 2005.

17.- Organización Mundial de la Salud. Trastornos mentales y del comportamiento. Descripciones clínicas y pautas para el diagnóstico CIE-10. España: Meditor; 1992.

18.- Scharko AM. DSM psychiatric disorders in the context of pediatric HIV/AIDS. AIDS Care 2006; 18 (5): 441-5.

19.- Xu T, Yan Z, Duan S, Wang Ch, Rou K, Wu Z. Psychosocial well-being of children in HIV/ AIDS-affected families in Southwest China: A qualitative study. J Child Fam Stud 2009; 18: 21-30.

20.- Freeman M. Patel V, Collins P Y, Bertolote J. Integrating mental health in global initiatives for HIV/AIDS. Br J Psych 2005; 187: 1-3.

21.- Nozyce M L, Lee S S, Wiznia A, Nachman S, Mofenson L M, Smith M E, et al. A behavioral and cognitive profile of clinically unstable HIV-infected children. Pediatrics 2006; 117 (3): 763-70.

22.- Chernoff M, Nachman S, Williams $P$, Brouwers P, Heston J, Hodge J, et al. IMPAACT P1055 Study Team. Mental health treatment patterns in perinatally HIV-infected youth and controls. Pediatrics 2009; 124: 627-36. Disponible en: http://www.pediatrics. org/cgi/content/full/124/2/627. (Accedido: 15 de febrero del 2010).

23.- Abubakar A, Van Baar A, Van de Vijver F J R, Holding P, Newton C R J. Pediatric HIV and neurodevelopment in sub-Saharan Africa: a systematic review. Trop Med Int Health 2008; 13 (7): 880-7.

24.- Naudé D, Pretorius R. Proposing an instructional framework for children with HIV/ AIDS. Br J Special Educ 2003; 30 (3): 138-43.

25.- Skovdal M, Ogutu V O, Aoro C, Campbell C. Young careers as social actors: Coping strategies of children caring for ailing or ageing guardians in Western Kenya. Soc Sci Med 2009; 69: 587-95.

26.- Mellins C A, Smith R, O’Driscoll P, Magder L S, Brouwers P, Chase C, et al. High rates of behavioral problems in perinatally HIVinfected children are not linked to HIV disease. Pediatrics 2003; 111: 384-93.

27.- Bagenda D, Nassali A, Kalyesubula I, Sherman B, Drotar D, Boivin M J, et al. Health, neurologic, and cognitive status of HIV-infected, long-surviving, and antiretroviralnaive Ugandan children. Pediatrics 2006; 117: 729-40.

28.- Wacharasin C, Homchampa P. Uncovering a family care giving model: insights from research to benefit HIV-infected patients, their caregivers, and health professionals. J Assoc Nurses AIDS Care 2008; 19 (5): 385-96. 